ISSN 2306-1561

Automation and Control in Technical Systems (ACTS)

2014, No 1.2(9), pp. 44-60.

DOI: $10.12731 / 2306-1561-2014-1-18$

\title{
Analysis of existing approaches in developing Decision Support Systems: Ontology and Mivar Nets
}

\section{Chibirova Marina Olegovna}

Russian Federation, Junior Researcher.

Scientific-Research Institute "MIVAR" (SRI "MIVAR"), 127572, Russian Federation, Moscow, Altufevskoe Highway, 95 B. http://www.mivar.ru

\section{mashkache@gmail.com}

\begin{abstract}
The aim of this paper is researching decision support systems (DSS) and existing approaches in building them. Limitations and advantages of using each of the methods in all phases of the DSS have been analyzed, as well computational complexity of the tasks. Here is an approach allows simulating, accumulating and logical processing large amounts of data. This will improve intelligence of decision support systems, expand the boundaries of tasks and create a new generation of mivar DSS.
\end{abstract}

Keywords: mivar, mivar nets, decision support systems (DSS), ontology, decisions based on precedents, logical systems with linear computational complexity. 
ISSN 2306-1561

Автоматизация и управление в технических системах (АУТС) 2014. - №1.2(9). - C. 44-60.

DOI: $10.12731 / 2306-1561-2014-1-18$

\section{УДК 004.8}

\section{Анализ подходов к построению систем поддержки принятия решений: Онтологии и Мивары}

\section{Чибирова Марина Олеговна}

Российская Федерация, младший научный сотрудник.

Научно-исследовательский институт «МИВАР» (НИИ «МИВАР»), 127572, Российская Федерация, Москва, Алтуфьевское ш., д. 95 Б. http://www.mivar.ru

mashkache@gmail.com

Аннотация. В работе исследованы два подхода к созданию систем поддержки принятия решений (СППР): онтологии и мивары. Выделяются недостатки и достоинства использования каждого из методов на всех этапах разработки СППР, анализируется сложность решаемых задач. Предложено использовать миварный подход, позволяющий моделировать, накапливать и логически обрабатывать большие объемы данных. Обосновано, что онтологии являются частным случаем миварных сетей. Применение миваров позволит повысить интеллектуальность систем поддержки принятия решений, расширить границы рассматриваемых задач и создать СППР нового поколения.

Ключевые слова: мивар, миварные сети, системы поддержки принятия решений (СППР), онтологии, принятие решений на основе прецедентов, логический вывод с линейной вычислительной сложностью.

\section{1. Введение}

Развитие информационных систем и технологий обуславливает необходимость создания и применения различных программных систем поддержки принятия решения (СППР). СППР состоят из двух основных компонент: хранилища данных и аналитических средств. В качестве хранилища данных могут выступать модели предметных областей, различные документы и базы знаний. Аналитические средства СППР предназначены для оказания помощи в принятии решений на основе использования этих данных, в зависимости от типа которых СППР можно разделить на оперативные и стратегические. Оперативные СППР предназначены для немедленного 
реагирования на текущую ситуацию, а стратегические основаны на анализе информации из разных источников с привлечением сведений, содержащихся в системах, аккумулирующих опыт решения проблем.

СППР могут опираться на различные методы принятия решений. Выделяют три основных этапа их разработки [1]:

- извлечение и представление знаний;

- принятие решений;

- построение «человеко-машинных» (диалоговых) систем.

Прежде всего, необходимо описать предметную область, т.к. чем больше информации о рассматриваемой ситуации вовлечено в процесс принятия решений, тем более обоснованный вывод будет получен. Как известно, существуют различные подходы, методы и языки представления данных [1 - 36]. В настоящее время наиболее широкое применение находят онтологии, однако предложен еще один подход миварное пространство. Отметим, что проведенный в работе [36] сравнительный анализ когнитивных карт и миварного подхода, показал миварный подход обобщает когнитивные карты, которые могут рассматриваться как частный случай миварных сетей.

В данной работе продолжим тему сравнения миваров с известными («традиционными») моделями представления знаний и проанализируем онтологии.

\section{2. Анализ онтологического подхода}

Онтология представляет собой совокупность терминов и взаимосвязанных определений, относящихся к некоторой предметной области и выполняющих нормативную функцию. Именно онтология формирует самое общее представление об объекте исследования [2]. Будем говорить, что онтология предметной области - это кортеж вида

$$
O^{d}=\langle C, A, R, D\rangle
$$

где $C$ - множество понятий предметной области, $A-$ множество их атрибутов, $R-$ отношение частичного порядка на множестве понятий, $R \subseteq C \times C, D-$ множество областей допустимых значений атрибутов. Понятие определяется как $\left\langle d_{i}, F\left(d_{i}\right), V\left(d_{i}\right)\right\rangle$, где $d_{i}$ - имя понятия, $F\left(d_{i}\right)=\left\{f_{i j}\right\}$ - множество признаков понятия, $V\left(d_{i}\right)=\left\{v_{i q}\right\}$ - объем понятия, viq - объекты, имеющие признаки $F\left(d_{i}\right)$.

Пусть $B(F(d i))=\left\{\varnothing, 2^{|F(d)|}\right\}$ - булеан содержания $F\left(d_{i}\right)$ некоторого понятия $d_{i}$, где $2^{|F(d)|}$ - множество всех подмножеств содержания $F\left(d_{i}\right)$. Элементы булеана образует частично упорядоченное множество по включении его элементов, т.е. решетку $\left(B\left(F\left(d_{i}\right)\right) \leq, \wedge, \quad \vee\right)$ 
Любой элемент $F\left(d_{i}{ }^{H}\right) \in B\left(F\left(d_{i}\right)\right), H \in\left[1,2^{|F(d)|}\right]$ полученной решетки формально будем считать содержанием некоторого понятия $d_{i}^{H}$ обобщающего понятие $d_{i}$, если $F\left(d_{i}{ }^{H}\right) \subseteq F\left(d_{i}\right)$. Решетку $K\left(d_{i}\right)=\left(B\left(F\left(d_{i}\right)\right) \leq, \Lambda, v\right)$ всех подмножеств содержания начального понятия $d_{i}$ будем называть концептуальным «каркасом» онтологии предметной области, в котором определены: абстрактные имена понятий $d_{i}^{H}$; множество признаков понятия $F\left(d_{i}{ }^{H}\right)$; отношения частичного порядка на множестве всех подмножеств содержания понятия $F\left(d_{i}\right)$ [4].

Стоит отметить, что понятия могут делиться на ряд классов:

- видовые и родовые понятия;

- понятия части и целого;

- единичные и общие;

- конкретные и абстрактные.

Отношения же представляют тип взаимодействия между понятиями предметной области. Формально n-арные отношения определяются как подмножество произведения $\mathrm{n}$ множеств: $R \subseteq C_{1} x C_{2} x \ldots x C_{n}$. Пример бинарного отношения отношение «часть-целое». Функции - это специальный случай отношений, в которых nй элемент отношения однозначно определяется n-1 предшествующими элементами. Формально функции определяются следующим образом:

$$
B: C_{1} \times C_{2} \times \ldots \times C_{n-1}->C_{n}
$$

Функциональные отношения определяются отношениями, свойственными множеству элементов. Иными словами, связи между элементами организуются с помощью именованных отношений вида: «быть частью», «принадлежать множеству», «совпадать с», «находиться в семантическом отношении с».

Процесс анализа и построения онтологии состоит из следующих этапов:

1) формулировка задачи и цели исследования;

2) изучение процесса с позиций поставленной цели;

3) сбор, систематизация и анализ существующих данных;

4) выделение объектов предметной области, для которых строится «каркас». Такие объекты должны иметь большее число признаков, по сравнению с остальными;

5) построение «каркаса» онтологии предметной области;

6) определение экспертом реальности обобщенных понятий $d i^{H}$ (класс) по их содержанию $F\left(d_{i}{ }^{H}\right)$ и имени необобщенного понятия $d$ (подкласс).

Итак, онтология понимается как структурная спецификация предметной области, включающая термины этой области, их свойства и логические связи, которые описывают их соотношения друг с другом. Она позволяет четко представить знания экспертов. Однако, такой подход довольно трудоемкий. 
Перейдем теперь к процессу принятия решений в онтологиях. Рассмотрим случай, когда решение принимается с целью разрешения какой-либо проблемы. Процесс разрешения проблемы состоит из трех стадий (рисунок 1) [3].

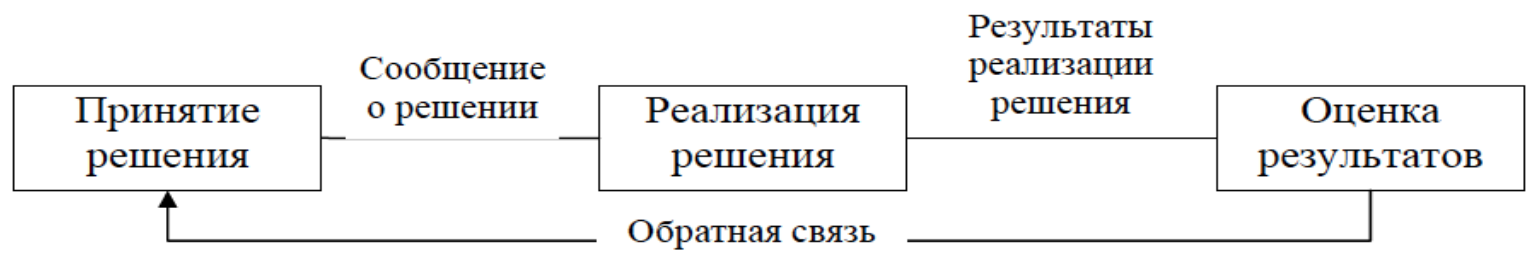

Рисунок 1 - Стадии разрешения проблем

В общем виде процесс принятия решений состоит из следующих основных этапов:

- выявление и диагностика проблемной ситуации;

- постановка цели;

- анализ факторов;

- разработка альтернатив;

- оценивание и выбор альтернатив.

Анализ текущей ситуации необходим для выявления симптомов проблемной ситуации. Он может быть реализован несколькими методами: интеллектуальный анализ данных, рассуждения на базе прецедентов, нейронные сети, компьютерное имитационное моделирование, OLAP-средства. После выявления проблемы происходит постановка цели, достижение которой может полностью решить проблему. Если в процессе анализа было обнаружено несколько причин возникновения проблемной ситуации, то каждая причина порождает отдельную задачу. После выявления причин проблемных ситуаций и постановки цели рассматриваются задачи выработки альтернатив их решения. После чего оценивание и выбор альтернатив происходит на основе экспертного мнения или некоторых критериев.

Согласно Н. Ною [6] существует ряд областей применения онтологий:

- для совместного использования людьми или программными агентами общего понимания структуры информации;

- для возможности повторного использования знаний в предметной области;

- для того чтобы сделать допущения в предметной области явными;

- д для отделения знаний в предметной области от оперативных знаний;

- д для анализа знаний в предметной области.

Построение онтологий часто не является конечной целью, обычно они используются другими программами для решения поставленных задач. К таким задачам, согласно С.Ниренбургу и В.Раскину [7], относится:

- машинный перевод;

- вопросно-ответные системы;

- информационный поиск;

- системы извлечения знаний; 
- системы понимания языка (автоматическое реферирование текста и др.).

Онтологические модели производственных систем являются основой использования аналитических методик в СППР. Анализ моделей предлагается осуществлять с помощью следующих основных методов, которые могут быть дополнены для решения специальных задач.

1. Визуализация моделей, отвечающая требованиям интерактивности и представляющая большие сети взаимосвязанных объектов, описывающих производственную систему, в наглядном виде, обеспечивающем удобное обозрение, навигацию, редактирование моделей и являющимся необходимым условием эффективного и качественного принятия решения.

2. Исследование моделей на основе графовых методик, т.е. анализ сетей объектов методами и средствами теории графов.

3. Фильтрация моделей и обеспечение различных режимов доступа пользователей к знаниям, заложенным в СППР. Метод реализуется за счет использования концептуальной информации о производственных системах, заложенной в онтологиях и предполагает возможность персонифицированной настройки отображения модели.

4. Манипуляция моделями, т.е. применение методов и средств, реализующих принцип многомодельности и объединяющих множество моделей, описывающих предметную область в различных аспектах, в единое информационное пространство. Для этого предлагается строить специальные информационные модели - приложения, реализующие идею «многоликих объектов» задачи, или акторов, лица которых представлены в различных объектных моделях. Благодаря этому подходу, становится возможным интеграция разноаспектных знаний об изучаемой системе и создание различных аналитических сводов информации для получения пользователем релевантной информации и ее использования для принятия эффективного решения.

5. Использование методики информационных запросов к моделям и поиска объектов. Предлагается организовывать взаимодействие с пользователем посредством интеллектуальных форм, управляемых на основе метаонтологии и онтологий производственных систем.

В частности, для осуществления информационных запросов к моделям вводится понятие коллекции объектов - группы объектов, обладающих общими заданными свойствами. В исследуемых информационно-логических моделях уже изначально существуют стандартные коллекции объектов, а произвольный запрос пользователя приводит к формированию новых коллекций, удовлетворяющих некоторым задаваемым ограничениям. Ответом на запрос является фрагмент объектной модели, причем объекты доступны со всеми их свойствами и связями.

Конструирование запросов-ограничений предлагается осуществлять на основе логики предикатов первого порядка в форме логических дескрипций. На основе использования метаонтологии и онтологий разработана классификация логических 
дескрипций и предложено формирование соответствующих видов информационных запросов, реализующихся в соответствующих интеллектуальных интерактивных формах взаимодействия с пользователем [2].

В этих случаях к онтологии формируется запрос. Слова запроса соединяются логическими связями: «и», «или», «не». Таким образом, запрос представляется логической формулой, которая может содержать термины $(T 1, T 2, \ldots, T N)$ и ограничения (дополнительные условия). Если существует база, содержащая множество документов $\left(D_{0}\right)$, по которым осуществляется поиск, то каждому термину сопоставляется множество документов, содержащих этот термин $\left(D_{T}\right)$. Далее происходит объединение, пересечение и дополнение этих множеств:

$$
\begin{aligned}
& T 1 \vee T 2 \sim D_{T 1} \cup D_{T 2}, \\
& T 1 \& T 2 \sim D_{T 1} \cap D_{T 2}, \\
& \neg T \sim D_{0} \backslash D_{T}
\end{aligned}
$$

Ответом на запрос является документ в общем или фрагмент объектной модели, причем объекты доступны со всеми их свойствами и связями.

У такого подхода есть множество недостатков. Например, на запрос может вернуться много документов или поиск будет проходить по всем словам, составляющим формулы. Слова имеют одинаковую важность (вес), хотя могут быть ключевым, а могут лишь дополнять описания. Для устранения первой проблемы можно задать сложную логическую формулу поиска, но для этого нужно хорошо разбираться в предметной области. Так же можно использовать ранжированный поиск. Он позволяет выделить релевантные документы через распределение частот встречаемости терминов запроса в документах и требует дополнительной обработки данных.

В случае использования онтологии лишь в качестве описания предметной области исследование моделей осуществляется на основе графовых методик, т.е. анализ сетей объектов методами и средствами теории графов. В этом случае алгоритмы выбираются с участием эксперта, имеют высокую вычислительную сложность и не вариативны. Это также накладывает ограничения на диапазон решаемых задач и полноту их описания.

Если же стоит вопрос решения более сложных задач, то основными модулями будут являться не только онтологическое описание знаний и алгоритм поиска на основе онтологий, но и онтология прецедентов.

Прецедент - это описание проблемы или ситуации в совокупности с подробным указанием действий, предпринимаемых в данной ситуачии для решения данной проблемы [8] (накопленный опыт экспертов по решению проблем).

Для интеллектуальной поддержки принятия решений на основе прецедентов проблемных ситуаций используются три типа правил:

1) правила распознавания класса прецедентов $R^{R e c}$, к которому следует отнести текущую проблемную ситуацию; 
2) правила пополнения запроса $R^{C}$, которые выводят дополнительные признаки из известных признаков, содержащихся в запросе пользователя, в соответствии с распознанным классом проблемной ситуации;

3) правила адаптации $R^{A}$, которые описывают, как решение, извлеченное из найденного ближайшего прецедента, может быть адаптировано к новому запросу.

Задача принятия решений $d^{P S}$ на основе множества решений, содержащихся в найденных k ближайших прецедентах, представима кортежем следующего вида:

$$
d^{P S}=<D^{\text {Case }}, F, \operatorname{Sim}^{X}, M, R^{A}>\text { с учетом } \xi=\{\xi 1, \ldots, \xi S\}
$$

где $D^{\text {Case }}$ - множество альтернатив решений, содержащихся в найденных $k$ ближайших прецедентах;

$F$ - множество критериев оценки альтернатив решений;

$\operatorname{Sim}^{X}$ - множество значений сходства между признаками проблемной ситуации и соответствующими признаками прецедентов;

$M$ - множество методов, позволяющих получить отображение множества альтернатив в множество векторных оценок эффективности решений в соответствии с множеством критериев $F$;

$R^{A}$ - множество решающих правил адаптации, устанавливающих отношения между значениями сходства, полученными в результате сопоставления признаков проблемной ситуации и признаков прецедентов и действиями, корректирующими атрибуты классов решений;

$\xi$ - множество $S$ типов вариантов среды решения задачи (детерминированная, вероятностная, нечеткая и др.).

Вывод, основанный на прецедентах, особенно эффективен, когда основным источником знаний о задаче является опыт, а не теория, решения не уникальны для конкретной ситуации и могут быть использованы в других случаях, целью является не гарантированное верное решение, а лучшее из возможных [9].

Таким образом, СППР на основе онтологий являются скорее стратегическими, нежели оперативными системами. Они способны привлечь и использовать ранее полученные прецеденты, но медленно справляются с оперативным поиском решения.

\section{3. Анализ миварного подхода}

Перейдем теперь к рассмотрению миварного подхода, который базируется на самоорганизующихся эволюционных базах данных и правил [10 - 12]. Отметим, что основными преимуществами миварного подхода являются линейный матричный метод поиска логического вывода на адаптивной сети правил [13 - 14] и эволюционное миварное пространство [15] с возможностью параллельной обработки данных [16 - 17]. Миварный подход применяется для решения самых различных задач, включая 
самоорганизующиеся комплексы диагностики $[10,18]$, управление мобильными роботами [19], создание интеллектуальных систем [20, 21], информационная безопасность [22], распознавание образов [23] и др. Теоретически обоснованы $[24,25]$ и практически реализованы некоторые миварные интеллектуальные системы [26 - 35]. Направление создания миварных систем активно развивается. Например, в 2013 году опубликовано более 8 научных статей [28 - 35], а в 2014 была опубликована статья, содержащая сравнительный анализ возможностей когнитивных карт и миварных сетей для построения систем поддержки принятия решений [36].

В связи с тем, что подробное описание миварного подхода по аналогичному вопросу (сравнительному анализу с когнитивными картами) приведено в работе [36], приведем только краткое описание миваров, необходимое для целостности понимания данной работы.

Как известно, миварное пространство представляет собой множество осей, множество элементов осей, множество точек пространства и множество значений точек. Введем $A=\left\{a_{n}\right\}, n=1 . . N$, где $A$ - множество названий осей миварного пространства, $N$ - количество осей миварного пространства. Тогда $\forall a_{n} \exists F_{n}=\left\{f_{n_{n}}\right\}, n=1 . . N, i_{n}=1 . . I_{n}$, где $F_{n}$ - множество элементов оси an,in- идентификатор элемента множества $F_{n}, I_{n}=\left|F_{n}\right|$. Множества $F_{n}$ образуют многомерное пространство: $M=F_{1} \times F_{2} \times \ldots \times F_{n} . \quad m=\left(i_{1}, i 2, \ldots, i_{N}\right), m \in M, m-$ точка многомерного пространства, $\left(i_{1}, i_{2}, \ldots, i_{N}\right)$ - координаты точки т.

Существует множество значений точек многомерного пространства М:

$$
C_{M}=\left\{c_{i 1, i 2, . ., i N} \mid i_{1}=1 . . I_{1}, i_{2}=1 . . I_{2}, . ., i_{n}=1 . . I_{N}\right\}
$$

где $c_{i 1, i 2, . ., i N}$ - значение точки многомерного пространства $M$ с координатами $i_{1}, i_{2}, \ldots, i_{N}$. Для каждой точки пространства $M$ не существует или существует единственное значение из множества $C_{M}$. Таким образом, $C_{M}$ - множество изменений состояний модели данных, представляемой в многомерном пространстве. Для перехода между многомерным пространством и множеством значений точек введено отношение $\mu: C_{x}=\mu\left(M_{x}\right)$, где $M_{x} \subseteq M, M_{x}=F_{1 x} \times F_{2 x} \times \ldots \times F_{N x}$.

Миварная технология накопления знаний - это способ создания глобальных эволюционных баз знаний $\mathrm{c}$ изменяемой структурой на основе адаптивного дискретного миварного информационного пространства представления данных. Миварное информационное пространство образуется тремя осями: ось отношений $O$, ось признаков (свойств) элемента $S$ и ось элементов (объектов) предметной области $V$ (рисунок 2) [36]. 


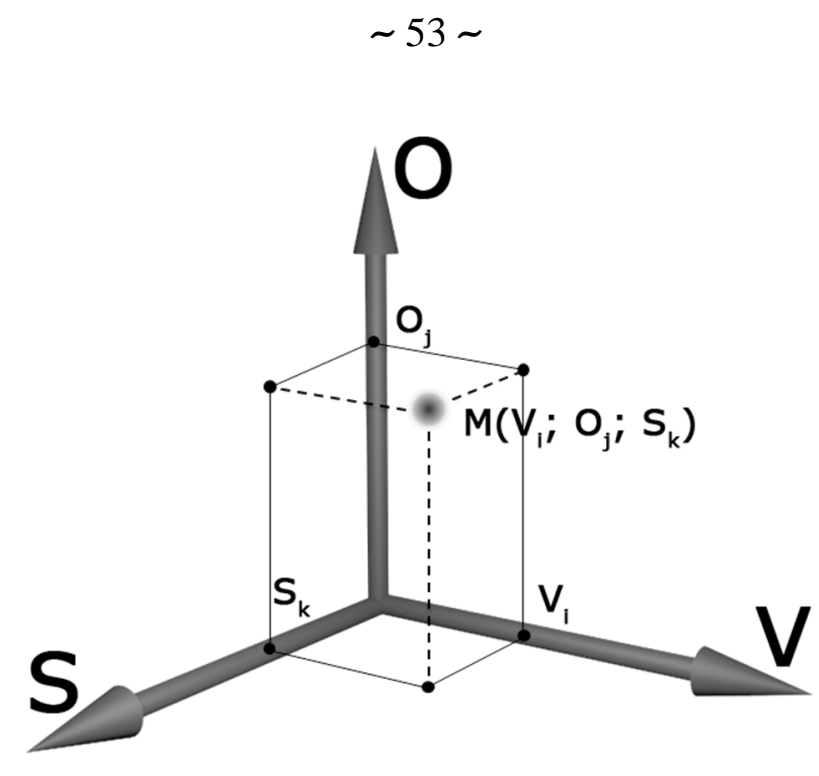

Рисунок 2 - Трехмерное пространство представления данных

Элементы связаны друг с другом отношениями. Множество всех точек многомерного пространства соответствует модели данных.

В целях проведения корректного сравнительно анализа, проведем формализацию миварного подхода по аналогии с онтологиями.

Таким образом, получаем, что аналогично кортежу онтологий (1), кортеж миварного пространства можно выразить следующим образом:

$$
\langle C, A, R, D, S\rangle
$$

где $\mathrm{C}$ - множество понятий предметной области, $A$ - множество их атрибутов, $R$ - отношение частичного порядка на множестве понятий, $R \subseteq C \times C, D$ - множество областей допустимых значений атрибутов, $S$ - множество признаков понятий.

Элементы миварного пространства, аналогично элементам онтологии, могут быть связаны отношениями «общее - частное», «конкретное - абстрактное» или быть объектами многоуровневой иерархии.

Кроме того, отношения могут быть представлены формулами, логическими переходами, текстовыми выражениями, функциями, сервисами и т.д. При этом в отношения может вступать неограниченное количество элементов, что обеспечивает полноту описания предметной области.

Процесс анализа и построения миварной модели предметной области состоит из следующих этапов:

- сбор существующих данных в рассматриваемой области;

- выделение и описание объектов, их признаков и отношений.

Напомним, как происходит процесс принятия решений на основе миварного подхода. Пусть известны $\mathrm{m}$ - правил и $\mathrm{n}$ - переменных (входящих в правила либо в качестве исходных, активизирующих, либо в качестве получаемых, т.е. выходных переменных). Тогда зададим двумерную матрицу $V(n \times m)$, в которой каждое правило 
будет содержать информацию о своих входных и выходных параметрах, а каждый объект, соответственно, о правилах, в которых он участвует, и своей в них роли. Это позволяет избежать перебора и, постепенно выявляя новые известные элементы через соответствующие правила, постоянно сокращать размерность исходной матрицы. Подробнее с методом поиска решений и формирования двумерной матрицы миварной сети можно ознакомится в научных публикациях [10 - 36].

Итак, миварный подход позволяет создавать многомерные базы данных, обрабатывать обрабатывает большие объемы данных в режиме реального времени с совмещением логических выводов и вычислительной обработки. Решения уникальны для каждой конкретной ситуации и строятся автоматически, без необходимости участия эксперта.

\section{4. Сравнение онтологий и миварного подхода}

Введем следующие графические обозначения:

\begin{tabular}{|ll|}
\hline & Понятие \\
\hline & Отношение \\
\hline & Свойства \\
\hline
\end{tabular}

Тогда в общем виде модель онтологий можно представить в виде графа или иерархии (рисунок 3). Элементы могут иметь несколько свойств, признаков объектов, возможность выражения отношений ограничена.

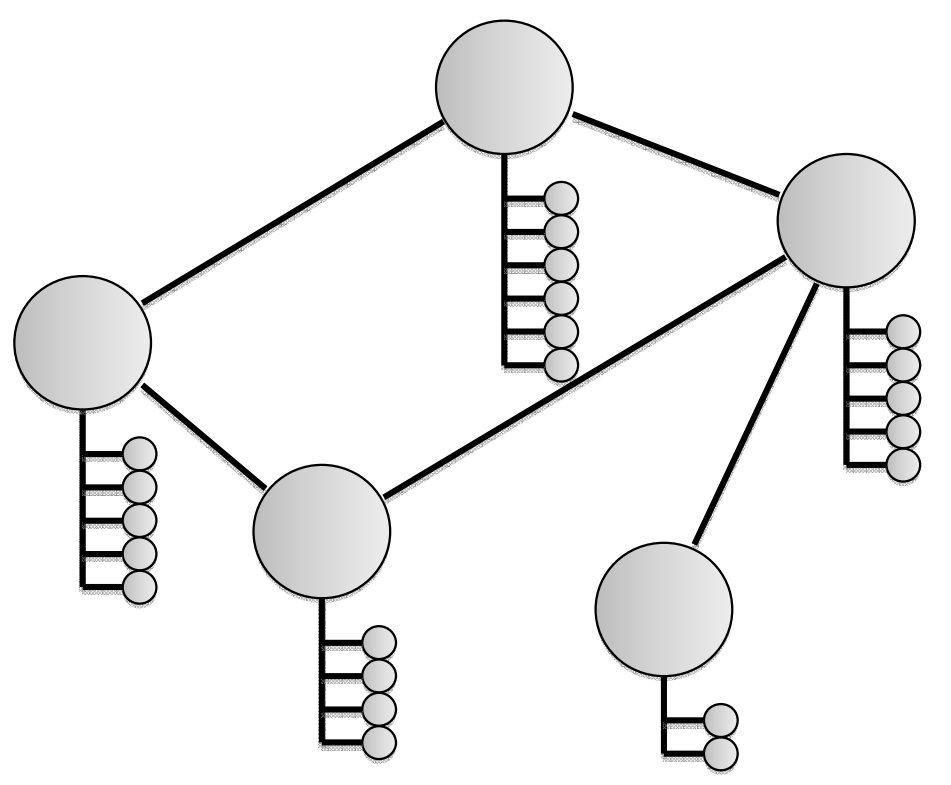

Рисунок 3 - Схематичное представление онтологий 
В подобном графическом формализме миварные сети можно отобразить в следующем виде (рисунок 4), который предложен в работе [36].

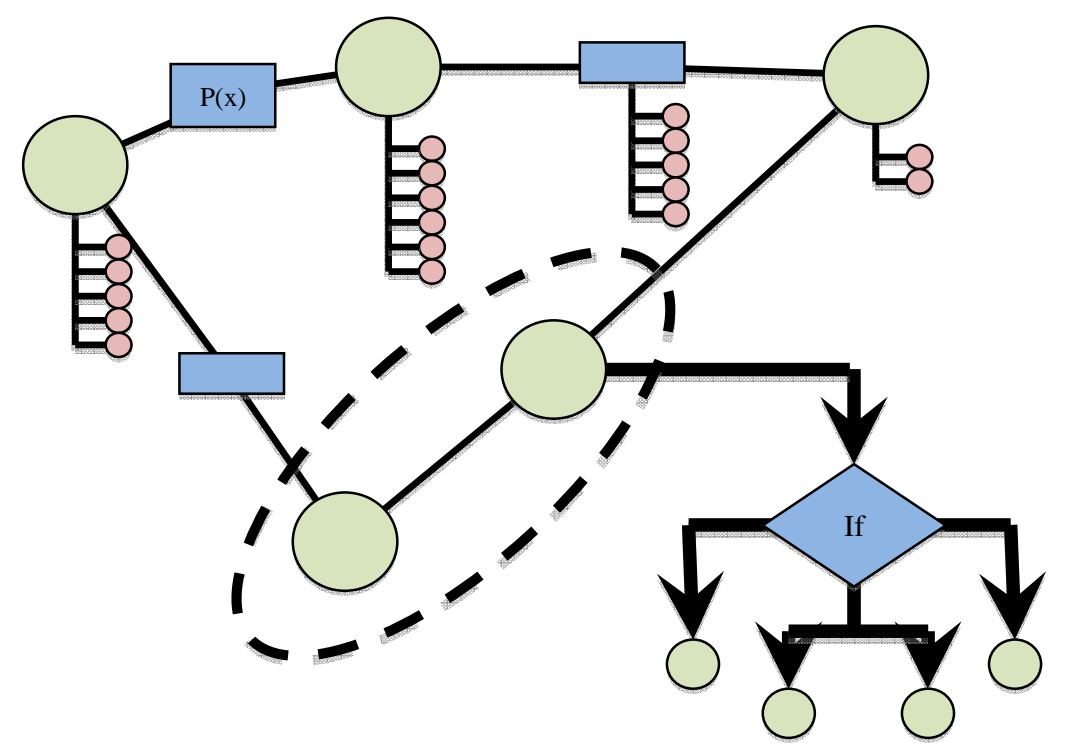

\section{Рисунок 4 - Схематичное представление миварного подхода}

Проведенный анализ показал, что в миварном подходе объекты и связи могут иметь неограниченное количество свойств. Возможность выражения отношений не ограничена, и способна учитывать любой набор входных и выходных элементов [1336]. Приведем все описанное в виде таблицы 1.

Таблица 1 - Сравнительный анализ онтологий и миварного подхода

\begin{tabular}{|c|c|c|}
\hline Параметры сравнения & Онтологии & Миварный подход \\
\hline $\begin{array}{l}\text { Предварительная } \\
\text { формулировка задачи } \\
\text { цели исследования }\end{array}$ & Требуется & Не требуется \\
\hline $\begin{array}{l}\text { Предварительное изучения } \\
\text { ситуации с точки зрения } \\
\text { поставленной цели }\end{array}$ & Требуется & Не требуется \\
\hline Сбор данных & $\begin{array}{c}\text { С учетом ограничений } \\
\text { контекста } \\
\text { рассматриваемой } \\
\text { ситуации }\end{array}$ & $\begin{array}{c}\text { Без учета ограничений } \\
\text { контекста рассматриваемой } \\
\text { ситуации }\end{array}$ \\
\hline $\begin{array}{l}\text { Объективность полученной } \\
\text { модели }\end{array}$ & - & + \\
\hline $\begin{array}{l}\text { Возможность } \\
\text { признаков элементов (их } \\
\text { свойств) }\end{array}$ & Есть & Есть \\
\hline
\end{tabular}




\begin{tabular}{|c|c|c|}
\hline Параметры сравнения & Онтологии & Миварный подход \\
\hline $\begin{array}{l}\text { Предварительный анализ и } \\
\text { формулировки } \\
\text { решения }\end{array}$ & Требуется & Не требуется \\
\hline Добавление данных & $\begin{array}{c}\text { Добавление нового } \\
\text { элемента в иерархию } \\
\text { может потребовать } \\
\text { изменение структуры }\end{array}$ & $\begin{array}{c}\text { Добавление нового элемента } \\
\text { приводит к автоматическому } \\
\text { изменению множества, } \\
\text { определяющего ось } \\
\text { пространства }\end{array}$ \\
\hline Изменение данных & $\begin{array}{c}\text { Происходит изменение } \\
\text { данных в иерархии, может } \\
\text { потребоваться изменение } \\
\text { структуры модели }\end{array}$ & $\begin{array}{c}\text { Результатом выполнения } \\
\text { является сохранение } \\
\text { значений новых точек в } \\
\text { многомерном пространстве }\end{array}$ \\
\hline $\begin{array}{l}\text { Описание отношения } \\
\text { между элементами }\end{array}$ & $\begin{array}{c}\text { Характерны отношения } \\
\text { типа: «быть частью», } \\
\text { «принадлежать } \\
\text { множеству», «совпадать } \\
\text { с», «находиться в } \\
\text { семантическом } \\
\text { отношении с» }\end{array}$ & $\begin{array}{c}\text { Широкие возможности по } \\
\text { описанию взаимодействия } \\
\text { объектов: формулы, } \\
\text { логические переходы, } \\
\text { текстовые выражения (а так } \\
\text { же внешние сервисы) }\end{array}$ \\
\hline $\begin{array}{l}\text { Возможность вариативного } \\
\text { представления данных } \\
\text { (классовое, иерархическое, } \\
\text { общее и т.д.) }\end{array}$ & Есть & Есть \\
\hline $\begin{array}{lr}\text { Последующее } & \text { изучение } \\
\text { описанной } & \text { модели, } \\
\text { определение } & \text { экспертом } \\
\text { реальности } & \text { обобщенных } \\
\text { понятий } & \\
\end{array}$ & Требуется & Не требуется \\
\hline $\begin{array}{l}\text { Алгоритм процесса } \\
\text { принятия решений }\end{array}$ & $\begin{array}{c}\text { Используется либо } \\
\text { алгоритм поиска по } \\
\text { дереву, либо поиск } \\
\text { прецедентов }\end{array}$ & $\begin{array}{c}\text { Разрабатывается каждый раз } \\
\text { автоматически }\end{array}$ \\
\hline Вариативность алгоритма & $\begin{array}{c}\text { Зависит от ситуации и } \\
\text { выбора метода принятия } \\
\text { решения }\end{array}$ & Вариативен \\
\hline $\begin{array}{l}\text { Время выполнения поиска } \\
\text { пути }\end{array}$ & $\begin{array}{c}\text { Экспоненциально } \\
\text { возрастает при } \\
\text { увеличении количества } \\
\text { рассматриваемых } \\
\text { элементов }\end{array}$ & $\begin{array}{c}\text { Выполняется в режиме } \\
\text { реального времени (линейная } \\
\text { вычислительная сложность) }\end{array}$ \\
\hline
\end{tabular}


На основе вышеизложенного приходим к выводу, что с помощью миварного подхода можно описать и выполнить все, что есть в онтологиях. Дополнительно к этому, в миварном подходе есть целый ряд дополнительных преимуществ. Таким образом, СППР на основе миварных сетей могут являться как стратегическими, так и оперативными системами. Следовательно, можно сказать: миварный подход обобщает и развивает онтологии, которые могут рассматриваться как частный случай миварных сетей.

\section{5. Заключение}

Проведен анализ использования двух подходов к разработке СППР. Процесс принятия решения был разделен на несколько этапов, на каждом из которых проводилось сравнение онтологий и миварного подхода. При использовании онтологий для разработки СППР необходимо:

- провести предварительный анализ рассматриваемой ситуации с учетом ограничений возможностей представления данных и видов связей;

- в случае добавления или изменений данных может потребоваться перестроение модели с участием эксперта;

- требуется проводить изучение описанной модели, определяя реальность обобщенных понятий;

- для поиска решения используется либо анализ сетей объектов методами и средствами теории графов, либо ищется аналогичный прецедент. В обоих случаях необходимо участие эксперта.

Однако онтологии дают возможность вариативно представлять данные (классы, иерархии и т.д.), описывать признаки объектов, задавать отношения типа «частьцелое», «принадлежать множеству» и т.д. Такие системы дают возможность сформировать и зафиксировать общие, разделяемые всеми экспертами знания о предметной области, наглядно отражают структуру модели и удобны при принятии решений на основе опыта. СППР на основе онтологий являются стратегическими системами. Они способны привлечь и использовать ранее полученные прецеденты, но медленно справляются с оперативным поиском решения.

Использование миварного подхода при разработке СППР не накладывает дополнительных ограничений на описание существующих знаний в данной предметной области. Элементы также могут быть связаны отношениями «часть-целое», «принадлежать множеству» и т.д. Кроме того, отношения могут быть выражены в виде формул, логических переходов, текстовых выражений, функций. При добавлении данных происходит автоматическое изменение множеств, определяющих оси пространства, а при изменении сохраняется значение новых точек в пространстве. В ходе решения сам миварный движок генерирует уникальные алгоритмы поиска пути в зависимости от исходных данных, после чего происходит расчёт необходимых выражений в режиме реального времени и вывод найденного алгоритма с отображением использованных объектов и связей между ними. 
Таким образом, миварный подход способен помочь в решении широкого спектра задач, наиболее полно описать предметную область и учесть ее особенности. СППР на основе миварных сетей могут являться как стратегическими, так и оперативными системами, способными найти решение в режиме реального времени, обосновать его правильность и произвести расчет, если это необходимо.

\section{Список информационных источников}

[1] Минин Д.В., Фролов С.В. Применение систем поддержки принятия решений при разработке медицинский программно-аппаратных комплексов. [Электронный pecypc]. URL: rgu-penza.ru/mni/content/files/ 10_1_Minin,Frolov.pdf (дата обращения: 06.12.2013).

[2] Сухов С.В. Онтология управления организациями // Журнал "Менеджмент в России и за рубежом" №5 год - 2003.

[3] Ситников П.В. Построение систем поддержки принятия решений на основе онтологий // Автореф. дис. канд. тех. наук / Институт проблем управления сложными системами. Самара. 2009.

[4] Кулинич А.А. Концептуальные «каркасы» онтологий в поддержке принятия решений в условиях неопределенности. [Электронный ресурс]. URL:http://raai.org/resurs/papers/kii-2012/doclad/Kulinich.doc (дата обращения: 06.12.2013).

[5] Добров Б.В., Иванов В.В., Лукашевич Н.В., Соловьев В.Д. Онтологии и тезаурусы: модели, инструменты, приложения / [Электронный ресурс]: учебный курс Интернет-университета информационных технологий. - Режим доступа: http://www.intuit.ru/department/expert/ontoth/2/

[6] Noy N., McGuinness D. L. Ontology Development 101: A Guide to Creating Your First Ontology. // Stanford Knowledge Systems Laboratory Technical Report KSL-01-05 and Stanford Medical Informatics Technical Report SMI-2001-0880, March 2001. //URL:http://protege.stanford.edu/

publications/ontology_development/ontology101.html

[7] Nirenburg S., Raskin V.. Ontological Semantics. Cambridge, MA, 2004.

[8] Джексон П. Введение в экспертные системы: Учеб. пособие. М.: Вильямс, 2001. $624 \mathrm{c}$.

[9] Черняховская Л.Р., Федорова Н.И., Низамутдинова Р.И. Интеллектуальная поддержка принятия решений в оперативном управлении деловыми процессами предприятия. Уфа: УГАТУ, 2011 Т. 15, № 2 (42). С. 172-176

[10] Варламов О.О. Эволюционные базы данных и знаний для адаптивного синтеза интеллектуальных систем. Миварное информационное пространство. - М.: Радио и связь, 2002. - 288 С.

[11] Варламов О.О. Системный анализ и синтез моделей данных и методы обработки информации в самоорганизующихся комплексах оперативной диагностики: диссертация на соискание ученой степени доктора технических наук. - М.: МАРТИТ, 2003. 307 с.

[12] Варламов О.О. Обзор 25 лет развития миварного подхода к разработке интеллектуальных систем и создания искусственного интеллекта // Труды НИИР. 2011. № 1. C. 34-44. 
[13] Варламов О.О. Разработка линейного матричного метода определения маршрута логического вывода на адаптивной сети правил // Известия вузов. Электроника. 2002. № 6. C. 43-51.

[14] Варламов О.О. Разработка адаптивного механизма логического вывода на эволюционной интерактивной сети гиперправил с мультиактивизаторами, управляемой потоком данных // Искусственный интеллект. 2002. № 3. С. 363-370.

[15] Варламов О.О. Основы многомерного информационного развивающегося (миварного) пространства представления данных и правил // Информационные технологии, 2003. № 5. С. 42-47.

[16] Варламов О.О. Разработка метода распараллеливания потокового множественного доступа к общей базе данных в условиях недопущения взаимного искажения данных // Информационные технологии. 2003. №1. С. 20-28.

[17] Варламов О.О. Параллельная обработка потоков информации на основе виртуальных потоковых баз данных // Известия высших учебных заведений. Электроника. 2003. № 5. С. 82-89.

[18] Варламов О.О. Системный анализ и синтез моделей данных и методы обработки информации для создания самоорганизующихся комплексов оперативной диагностики // Искусственный интеллект. 2003. № 3. С. 299-305.

[19] Варламов О.О. Системы обработки информации и взаимодействие групп мобильных роботов на основе миварного информационного пространства // Искусственный интеллект. 2004. № 4. С. 695-700.

[20] Варламов О.О. Создание интеллектуальных систем на основе взаимодействия миварного информационного пространства и сервисно-ориентированной архитектуры // Искусственный интеллект. 2005. № 3. С. 13-17.

[21] Варламов О.O. Анализ взаимосвязей GRID и CAC ИВК, SOA и миварного подхода // Искусственный интеллект. 2005. № 4. С. 4-11.

[22] Варламов О.О. О системном подходе к созданию модели компьютерных угроз и ее роли в обеспечении безопасности информации в ключевых системах информационной инфраструктуры // Известия Таганрогского государственного радиотехнического университета. 2006. Т. 62. № 7. С. 216-223.

[23] Максимова А.Ю., Варламов О.О. Миварная экспертная система для распознавания образов на основе нечеткой классификации и моделирования различных предметных областей с автоматизированным расширением контекста // Известия Южного федерального университета. Технические науки. 2011. № 12. С. 77-87.

[24] Варламов О.О. О необходимости перехода от теории искусственного интеллекта к разработке теории активного отражения // Известия Южного федерального университета. Технические науки. 2007. Т. 77. № 2. С. 89-95.

[25] Варламов О.О. О возможности создания интеллектуальных систем на основе GRID, систем адаптивного синтеза ИВК, сервисно-ориентированной архитектуры и миварного информационного пространства // Известия Таганрогского государственного радиотехнического университета. 2005. Т. 54. № 10. С. 130-140.

[26] Владимиров А.Н., Варламов О.О., Носов А.В., Потапова Т.С. Программный комплекс "УДАВ": практическая реализация активного обучаемого логического вывода с линейной вычислительной сложностью на основе миварной сети правил // Труды НИИР. 2010. Т. 1. С. 108-116.

[27] Варламов О.О. Миварные технологии: переход от продукций к двудольным миварным сетям и практическая реализация автоматического конструктора алгоритмов, управляемого потоком входных данных и обрабатывающего более 
трех миллионов продукционных правил // Искусственный интеллект. 2012.№4. С.11-33.

[28] Варламов О.О. Практическая реализация линейной вычислительной сложности логического вывода на правилах "ЕСЛИ-ТО" в миварных сетях и обработка более трех миллионов правил // Автоматизация и управление в технических системах. 2013. - № 1(3); [Электронный pecypc]. URL: http://auts.esrae.ru/3-66 (дата обращения: 26.03.2013).

[29] Варламов О.О., Чибирова М.О., Сергушин Г.С., Елисеев Д.В. "Облачная" реализация миварного универсального решателя задач на основе адаптивного активного логического вывода с линейной сложностью относительно правил "Если-То-Иначе" // Автоматизация и управление в технических системах. - 2013. - № 2(4). C. 7-23. [Электронный ресурс]. URL: auts.esrae.ru/4-78 (дата обращения: 26.05.2013).

[30] Варламов О.О., Сергушин Г.С., Елисеев Д.В., Адамова Л.Е., Майборода Ю.И., Антонов П.Д., Чибирова М.О. О миварном подходе к моделированию процессов понимания компьютерами смысла текстов, речи и образов. Новые возможности расширения границ автоматизации умственной деятельности человека. // Автоматизация и управление в технических системах. - 2013. - № 2(4); C. 30-45. [Электронный ресурc]. URL: auts.esrae.ru/4-80 (дата обращения: 26.05.2013).

[31] Сергушин Г.С., Варламов О.О., Чибирова М.О., Елисеев Д.В., Муравьева Е.А. Исследование возможностей информационного моделирования сложных систем управления технологическими процессами на основе миварных технологий // Автоматизация и управление в технических системах. - 2013. - № 2(4); С. 46-60. [Электронный ресурc]. URL: auts.esrae.ru/4-81 (дата обращения: 26.05.2013).

[32] Варламов О.О., Адамова Л.Е., Елисеев Д.В., Майборода Ю.И., Антонов П.Д., Сергушин Г.С., Чибирова М.О. Комплексное моделирование процессов понимания компьютерами смысла текстов, речи и образов на основе миварных технологий // Искусственный интеллект. - 2013. - № 4. - С. 15-27.

[33] Чибирова М.О., Сергушин Г.С., Варламов О.О., Елисеев Д.В., Хадиев А.М. и др. Реализация общедоступного миварного универсального решателя задач на основе адаптивного активного логического вывода с линейной сложностью и облачных технологий // Искусственный интеллект. - 2013. - № 3. - С. 512-523.

[34] Сергушин Г.С., Варламов О.О., Чибирова М.О., Елисеев Д.В., Муравьева Е.А. Информационное моделирование сложных автоматизированных систем управления технологическими процессами на основе миварных технологий // Искусственный интеллект. - 2013. - № 3. - С. 126-138.

[35] Варламов О.О., Чибирова М.О., Сергушин Г.С., Елисеев Д.В. Практическая реализация универсального решателя задач «УДАВ» с линейной сложностью логического вывода на основе миварного подхода и «облачных» технологий // Приборы и системы. Управление, контроль, диагностика. - 2013. - № 11. - С. 4555.

[36] Чибирова М.О. Сравнительный анализ возможностей когнитивных карт и миварных сетей для построения систем поддержки принятия решений // Автоматизация и управление в технических системах. - 2014. - № 1.1. С. 40-54. DOI: 10.12731/2306-1561-2014-1-5.URL: auts.esrae.ru/8-158 (дата обращения: 24.01.2014). 